\title{
The roles of TRIO and F-actin-binding protein in glioblastoma cells
}

\author{
HYUNJI LEE $^{1 *}$, MINHEE KIM ${ }^{1 *}$, JISOO PARK $^{1}$, QUANGDON TRAN $^{1}$, YOUNGEUN HONG $^{1}$, HYEONJEONG CHO $^{1}$, \\ SUNGJIN PARK ${ }^{1}$, SUNTAEK HONG ${ }^{2}$, DEREK P. BRAZIL ${ }^{3}$, SEON-HWAN KIM ${ }^{4}$ and JONGSUN PARK ${ }^{1}$ \\ ${ }^{1}$ Department of Pharmacology and Medical Science, Metabolic Syndrome and Cell Signaling Laboratory, Research Institute \\ for Medical Sciences, College of Medicine, Chungnam National University, Daejeon, Chungcheongnam-do 35015; \\ ${ }^{2}$ Laboratory of Cancer Cell Biology, Department of Biochemistry, School of Medicine, Gachon University, \\ Incheon, Gyeonggi-do 21999, Republic of Korea; ${ }^{3}$ Centre for Experimental Medicine, Queen's University \\ Belfast, Belfast BT9 7BL, UK; ${ }^{4}$ Department of Neurosurgery, Institute for Cancer Research, College of Medicine, \\ Chungnam National University, Daejeon, Chungcheongnam-do 35015, Republic of Korea
}

Received May 30, 2017; Accepted January 12, 2018

DOI: $10.3892 / \mathrm{mmr} .2018 .8458$

\begin{abstract}
TRIO and F-actin-binding protein (TrioBP), which was initially discovered as a binding partner of Trio and F-actin, is a critical factor associated with hearing loss in humans. However, the function of TrioBP in cancer has not been investigated. In the present study, TrioBP expression was indicated to be highly elevated in U87-MG and U343-MG cells. Furthermore, the TrioBP mRNA expression level was markedly increased in U87-MG and U251-MG cells compared with that in cerebral cortex cells, as determined by deep sequencing. Comprehensive analysis of a public TCGA dataset confirmed that TrioBP expression is elevated in patients with glioblastoma. In summary, the present data indicate that TrioBP expression is increased in glioblastoma cell lines and in patients with glioma, suggesting that TrioBP has potential as a diagnostic marker or therapeutic agent for glioma.
\end{abstract}

Correspondence to: Dr Seon-Hwan Kim, Department of Neurosurgery, Institute for Cancer Research, College of Medicine, Chungnam National University, 266 Munhwa-ro, Daejeon, Chungcheongnam-do 35015, Republic of Korea

E-mail: neons@cnu.ac.kr

Dr Jongsun Park, Department of Pharmacology and Medical Science, Metabolic Syndrome and Cell Signaling Laboratory, Research Institute for Medical Sciences, College of Medicine, Chungnam National University, 266 Munhwa-ro, Daejeon, Chungcheongnam-do 35015, Republic of Korea

E-mail: insulin@cnu.ac.kr

*Contributed equally

Key words: TrioBP, cytoskeleton, glioma, biomarker

\section{Introduction}

Gliomas, including glioblastoma multiforme (GBM), have a poor prognosis and high rate of relapse (1). The most common type of glial neoplasm is astrocytic tumor, approximately $80 \%$ of which are glioblastomas with common genetic defects (2). Gliomas can be treated by open surgery, chemotherapy, and/or radiotherapy. However, no effective treatments are available, resulting in a mean survival duration of $<15$ months (3).

Angiogenesis is a common feature of cancer and is mediated by upregulation of angiogenic genes (4). Angiogenesis leads to the development of large twisting vessels (5). The disordered vasculature of GBM leads to a diminished blood supply, which reduces the effectiveness of chemotherapy and radiotherapy (6). Anti-angiogenic approaches reportedly enhance the efficacy of chemotherapeutics (7). Bevacizumab (BEV), a recombinant protein that inhibits VEGF-A, is a promising candidate anti-angiogenesis drug (8). The efficacy of BEV cotreatment for GBM patients with temozolomide (TMZ) and radio/chemotherapy has been studied (9). A clinical study of avastin (AVAglio) in GBM patients indicated that cotreatment with $\mathrm{BEV}$ and avastin improved the progression-free survival duration (PFS, 4.4 months) while radiotherapy with BEV cotreatment showed no significant benefit for survival (10). Therefore, there is an urgent need to develop drugs other than TMZ and anti-VEGF agents for GBM patients.

TRIO and F-actin-binding protein (TrioBP) was identified as a novel Trio-interacting protein by screening of a skeletal muscle cDNA library (11). TrioBP contains an N-terminal pleckstrin homology domain and a C-terminal coiled-coil region. The interaction of TrioBP-1 with F-actin stabilizes the structure of the latter (11). Subsequent studies of the structure of TrioBP genes revealed that the TrioBP-4 and TrioBP-5 isoforms are required for hearing $(12,13)$. Linkage analysis of families has identified several mutations in the $22 \mathrm{q} 13$ region designated DFNB28, which spans 34 genes, including TrioBP-1 (14). TrioBP-1 is ubiquitously expressed, whereas TrioBP-4 and TrioBP-5 are expressed mainly in the eye 
and ear. Mutations in TrioBP-4 and TrioBP-5 have been linked to a form of autosomal recessive nonsyndromic deafness. All of the TrioBP mutations in DFNB28 that result in deafness are located in exon 6 of TrioBP and affect only the TrioBP-4 and TrioBP-5 isoforms (15). Because the siRNA-induced decrease in Trio expression inhibits the migration of glioblastoma cells (16), TrioBP, as a Trio-interacting protein, might be involved in the development of gliomas. Therefore, the role of TrioBP in glioblastoma was evaluated to enable the development of novel chemotherapeutics. Taken together, our results indicate that TrioBP has potential as a candidate therapeutic agent for glioblastoma. An enhanced understanding of the role of TrioBP in glioblastoma should provide important information for the management of cancer.

\section{Materials and methods}

Antibodies and reagents. Anti-TrioBP antibodies were purchased from Novus Biologicals, LLC (Littleton, CO, USA) and anti-actin antibodies were from Sigma-Aldrich (Merck KGaA, Darmstadt, Germany). Horseradish peroxidase-conjugated anti-mouse IgG or anti-rabbit IgG secondary antibodies were purchased from Komabiotech (Seoul, Korea). The siRNA against human TrioBP were synthesized by Intergrated DNA technologies. The siRNA sequences for TrioBP were the following; 5'-TCCCAGCAGAACCATCCAACAAGAGAA-3'.

Cell culture and transfection. The glioblastoma cells (U87, U25, and U343-MG) were maintained in medium (RPMI) supplemented with 10\% FBS, 25 mM HEPES (Thermo Fisher Scientific, Inc., Waltham, MA, USA), 1\% Antibiotics-Antimycotics (Thermo Fisher Scientific, Inc.). U87 and U251-MG cells were transiently transfected with $30 \mathrm{nM}$ control siRNA or TrioBP siRNA by using Neon Transfection System (Thermo Fisher Scientific, Inc.).

Patient samples. The study was approved by the Hospital Institutional Review Board (approval number CNUH 2013-11-006) according to the Declaration of Helsinki at Chungnam National University Hospital (Daejeon, Korea), and written informed consent was obtained from each patient before surgery. Normal brain tissue samples were obtained from cadavers alternatively, from autopsy of surrounding normal brain of glioblastoma patient who underwent surgery.

Immunoblot analysis. The immunoblot analysis was performed as the described previously $(17,18)$. Briefly, cells were placed on ice and extracted with lysis buffer containing $50 \mathrm{mM}$ Tris- $\mathrm{HCl}, \mathrm{pH} 7.5,1 \% \mathrm{v} / \mathrm{v}$ Nonidet P-40, $120 \mathrm{mM}$ $\mathrm{NaCl}, 25 \mathrm{mM}$ sodium fluoride, $40 \mathrm{mM} \beta$-glycerol phosphate, $0.1 \mathrm{mM}$ sodium orthovanadate, $1 \mathrm{mM}$ phenylmethylsulfonyl fluoride, $1 \mathrm{mM}$ benzamidine, and $2 \mu \mathrm{M}$ microcystin-LR. Lysates were centrifuged for $15 \mathrm{~min}$ at $12,000 \mathrm{x} \mathrm{g}$. The cell extracts were resolved by 10-15\% SDS-PAGE, and transferred to Immobilon-P membranes (EMD Millipore, Billerica, MA, USA). The filters were blocked for $1 \mathrm{~h}$ in $1 \mathrm{X}$ Tri-buffered saline buffer (TBS; $140 \mathrm{mM} \mathrm{NaCl}, 2.7 \mathrm{mM} \mathrm{KCl,} 250 \mathrm{mM}$ Tris- $\mathrm{HCl}$, pH 7.4), containing 5\% skimmed milk and $0.2 \%$ Tween-20, followed by an overnight incubation with the anti-TrioBP and anti-actin antibodies diluted 1,000 -fold at $4^{\circ} \mathrm{C}$. The secondary antibody was horseradish peroxidase-conjugated anti-mouse IgG or anti-rabbit IgG (Koma Biotech, Seoul, Korea), diluted 5,000 -fold in the blocking buffer. The detection of protein expression was visualized by enhanced chemiluminescence, according to the manufacturer's instructions (Thermo Fisher Scientific, Inc.).

Reverse transcription-quantitative reverse transcription-polymerase chain reaction ( $R T-q P C R)$. Total RNA was extracted from frozen tissue samples or from cells using the PureHelix RNA Extraction Solution (Nanohelix, South Korea). The cDNA was synthesized from total RNA with the SuperScript III First-Strand Synthesis System for RT-qPCR (Invitrogen; Thermo Fisher Scientific, Inc.). The RT-qPCR measurement of individual cDNAs was performed using SYBR-Green dye to measure duplex DNA formation with the StepOne Plus real-time PCR system (Invitrogen; Thermo Fisher Scientific, Inc.) and normalized to the expression of glyceraldehyde 3-phosphate dehydrogenase (GAPDH) RNA. The following primers were used in the RT-qPCR (forward, reverse); human TrioBP: F-5'-TCCAAGGTCTCCCTTAGTACA/R-5'-GTG GGACTGGACTTGCTA; human GAPDH: F-5'-TCGACA GTCAGCCGCATCTTCTTT/R-5'-TACGACCAAATCCGT TGACTCCGA.

RNA sequencing and RNA-Seq data analysis. Total RNA of U87-MG, U251-MG and normal brain was extracted using TRIzol reagent (Invitrogen) following the manufacturer's procedures. The total RNA quantity and purity were analysis of Bioanalyzer 2100 and RNA 6000 Nano LabChip Kit (Agilent Technologies, Inc., Santa Clara, CA, USA). Roughly $10 \mu \mathrm{g}$ of total RNA was subjected to isolate Poly (A) mRNA with poly-T oligo attached magnetic beads (Invitrogen; Thermo Fisher Scientific, Inc.). Following purification, the mRNA is fragmented into small pieces using divalent cations under raised temperature. Then the cleaved RNA fragments were reverse-transcribed to create the final cDNA library in accordance with the protocol for the mRNA-Seq sample preparation kit (Illumina, Inc., San Diego, CA, USA). The average insert size for the paired-end libraries was $300 \mathrm{bp}$ ( $\pm 50 \mathrm{bp}$ ). Next we performed the paired-end sequencing on an Illumina Hiseq 2000 system at Macrogen (Seoul, Korea) following the vendor's recommended protocol. For each sample, sequenced reads were aligned to the UCSC human reference genome (19) using the Tophat package (20), which initially removes a portion of the reads based on quality information accompanying each read and then maps the reads to the reference genome. FPKM (fragments per kilobase of exon per million fragments mapped) were calculated to compare the expression level of TrioBP mRNA variants in each sample.

Confocal imaging analysis and indirect immunofluorescence. U251-MG cells were grown on glass coverslips until they were $50-70 \%$ confluent. After $24 \mathrm{~h}$, the cells were fixed in $4 \%$ paraformaldehyde at room temperature for $10 \mathrm{~min}$ and permeabilized in $0.2 \%$ Triton $\mathrm{X}-100$ for $5 \mathrm{~min}$ at room temperature. Then cells were incubated in blocking buffer containing 5\% bovine serum albumin (Sigma-Aldrich; Merck KGaA) in $1 \mathrm{X}$ TBS for $1 \mathrm{~h}$ at $37^{\circ} \mathrm{C}$. The rabbit polyclonal anti-TrioBP was diluted 200 -fold for primary antibody and incubated for overnight. 
A
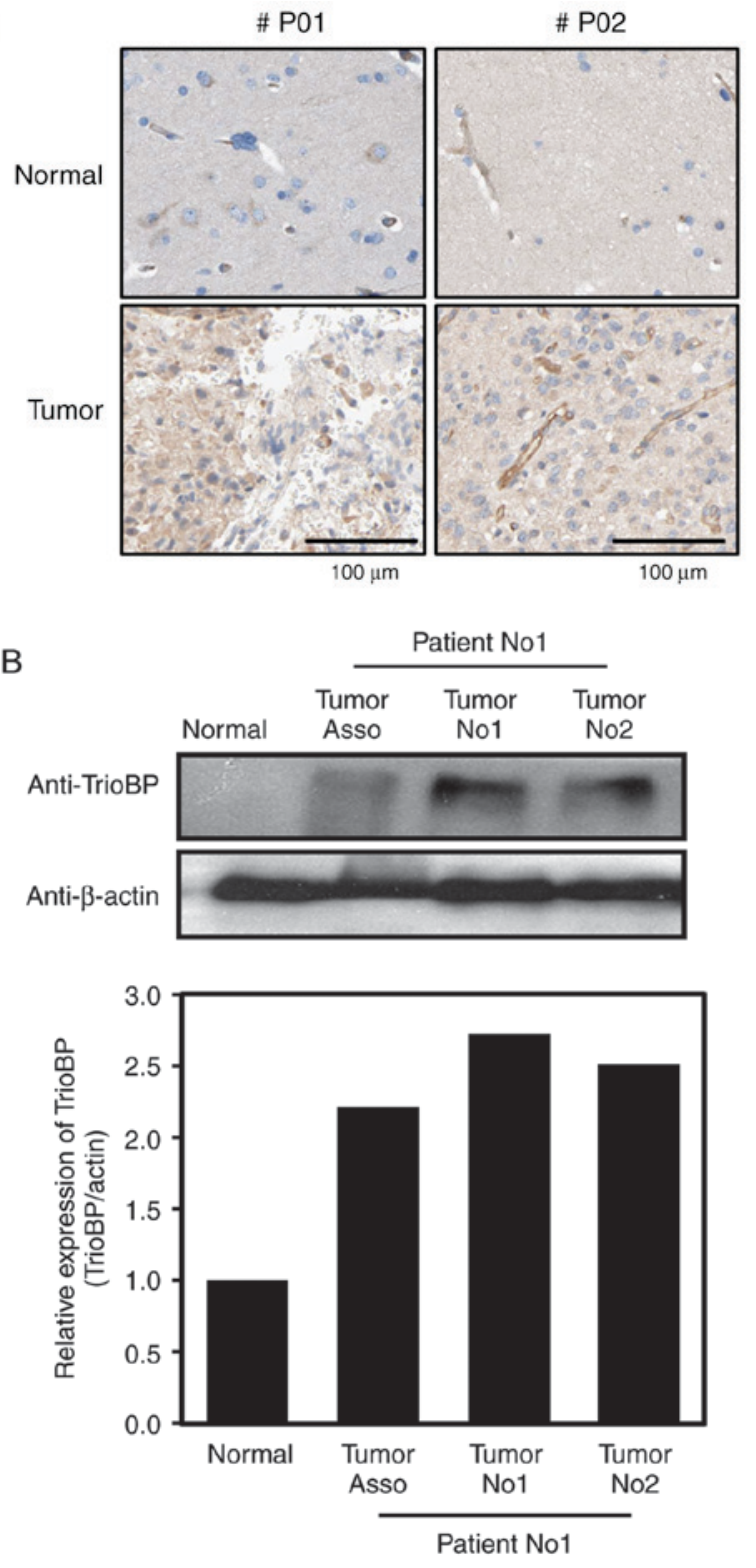

Figure 1. Changes of TrioBP expression in human brain tumors. (A) Human glioma tissue arrays were analyzed by immunohistochemistry (IHC) for TrioBP staining. Representative images from two patients were shown. Scale bars, $100 \mu \mathrm{m}$. (B) Total cell lysates from normal and GBM tissues (tumors no. 1 and 2) from patients were analyzed for TrioBP expression (top panel). Tumor-associated normal tissue served as a control (Tumor Asso.). Relative densities were calculated by densitometry. Relative differences in TrioBP expression levels were determined by normalizing all densitometric values to those of actin (in each lane) and setting the control values to 1 (bottom panel). The results are presented as means \pm SDs of data from two independent experiments. ${ }^{*} \mathrm{P}<0.05,{ }^{* *} \mathrm{P}<0.01$.

The secondary antibody, FITC-conjugated anti-rabbit antibody (BD Biosciences, Franklin Lakes, NJ, USA) was used. After appropriate rinsing, cover slips were mounted with Vectashield (Vector Laboratories, Inc., Burlingame, CA, USA) and visualized using a Zeiss confocal microscope (Zeiss AG, Oberkochen, Germany).

Immunohistochemistry. The analysis of immunohistochemistry was performed as the described previously (21). A human cancer tissue array slide with paraffin sections was purchased from Bio Max (US Biomax Inc; Thermo Fisher Scientific, Inc.).
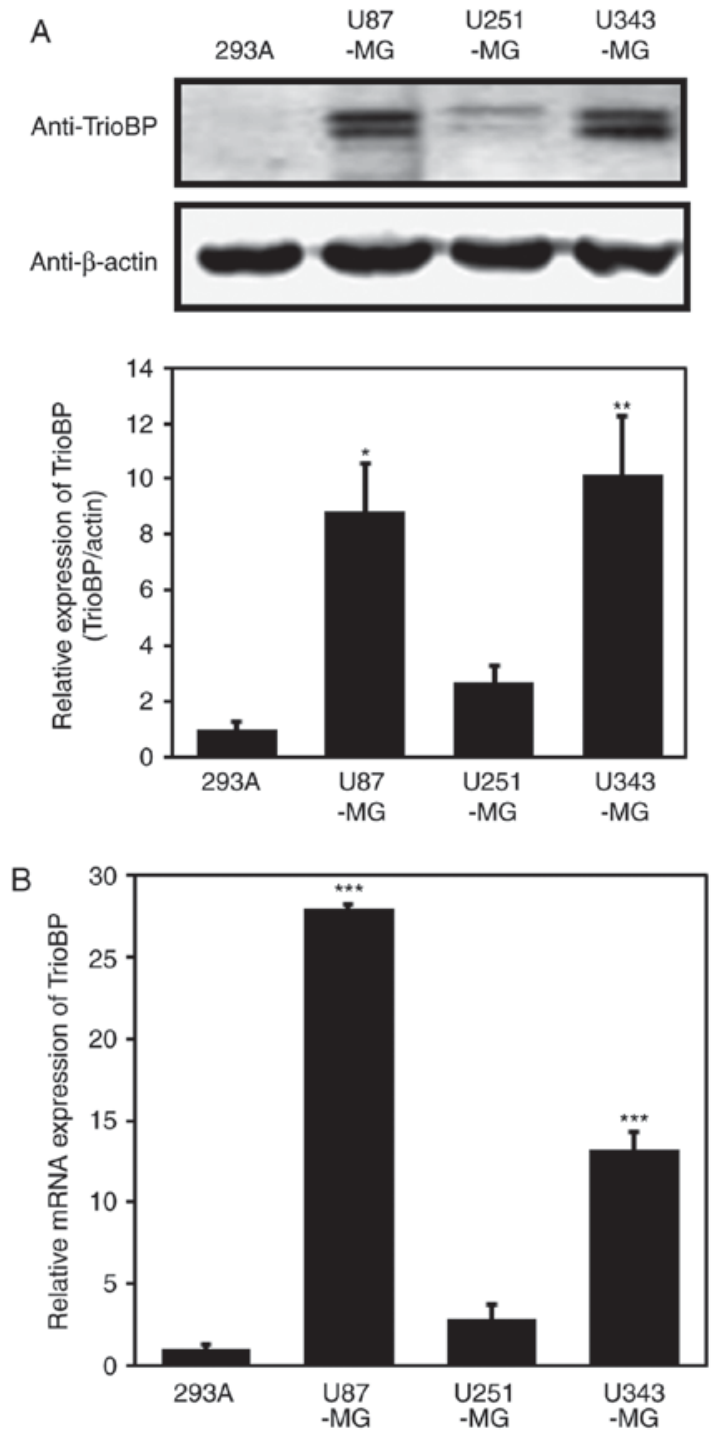

Figure 2. Expression of TrioBP in glioblastoma cell lines. (A) For immuno-blot analysis with anti-TrioBP and anti-actin antibodies, cell lysates were isolated from 4 established GBM cell lines (U87-MG, U251-MG, and U343-MG) and one established non-cancerous cell line (293A). The results are representative of 3-independent experiments (top panel). Relative density was obtained by densitometry of the corresponding immunoblot data. Relative and statistical differences of TrioBP expression were determined by normalizing values for actin in each lane and set the values for 293A as 1 (bottom panel). The results are presented as means \pm SD. of three independent experiments. Statistical analysis was performed using one-way ANOVA followed by Tukey's post hoc test $\left({ }^{*} \mathrm{P}<0.05,{ }^{* *} \mathrm{P}<0.01\right.$ vs. 293A). (B) Extracted total RNA from each GBM cell line was analyzed using human TrioBP specific primer sets by real-time quantitative reverse transcription-polymerase chain reaction (RT-qPCR), as described in the Materials and Methods section. The results are presented as means \pm SD of three independent experiments. Statistical analysis was performed using one-way ANOVA followed by Tukey's post hoc test $\left({ }^{* * *} \mathrm{P}<0.001\right.$ vs. $\left.293 \mathrm{~A}\right)$.

Histostain-Plus kits (Zymed Laboratories Inc., San Francisco, CA, US) were used in accordance with the manufacturer's instructions for the immunohistochemistry of tissue array. Briefly, paraffin sections were deparaffinized with xylene and rehydrated in a graded series of ethanol. The slide was submerged in peroxidase quenching solution for $10 \mathrm{~min}$. After it was washed twice with PBS for 5 min, it was added with 2 drops of Reagent A for blocking and incubated for $30 \mathrm{~min}$. Following two washes with PBS, the primary antibody was 


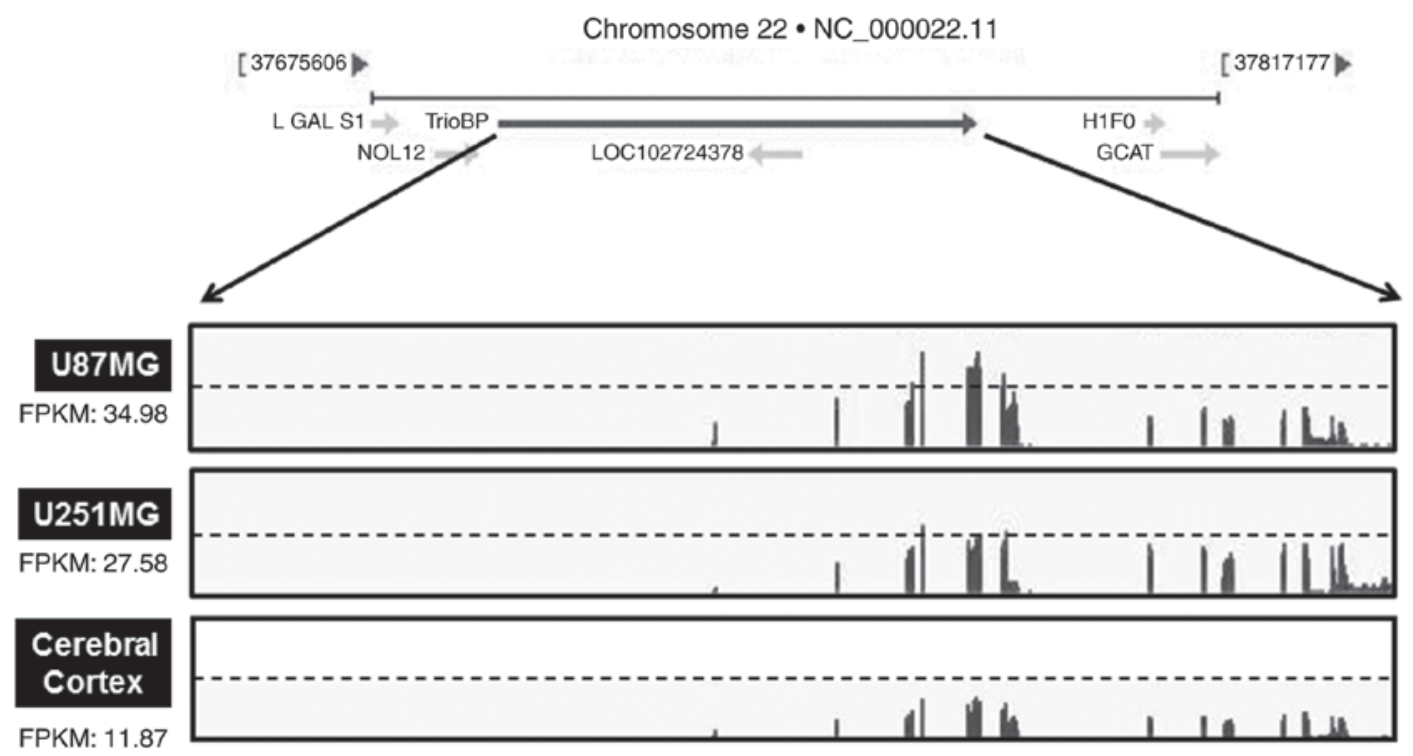

Figure 3. Relative changes of TrioBP transcripts from GBM cells in standard RNA-seq data. Total RNA were isolated from two GBM cell lines (U87-MG and U251-MG) and normal brain tissue. These samples were further analyzed by the standard RNA deep sequencing (RNA-seq) as described in the material and methods. RNA-seq read density for TrioBP transcripts was plotted with the relative RNA-seq read coverage (counts). Fragments per kilobase of exon per million fragments mapped (FPKM) were calculated to compare the expression level of TrioBP mRNA variants in each sample.

applied at $4^{\circ} \mathrm{C}$ for overnight. Then biotinylated secondary antibody, Reagent B, was added after rinsing with PBS. It was incubated at room temperature for $1 \mathrm{~h}$. It was rinsed with PBS and dropped with enzyme conjugated Reagent $\mathrm{C}$. After it was washed with PBS, DAB chromogen, and a mixture of Reagent D1, D2, and D3, it was dropped, and signals were observed with a florescence microscope. Then the reaction was stopped with distilled water, and pictures were taken with a microscope.

Bioinformatics data set. Glioma data sets and corresponding clinical data were downloaded from the publicly available databases (213 cases from the TCGA GBM dataset (http://www.betastasis.com/glioma/tcga_gbm/)). Normal; $\mathrm{n}=11$, Classical; n=54, Mesenchy-mal; $\mathrm{n}=58$, Neural; $\mathrm{n}=33$, Proneural; $\mathrm{n}=57$.

Real-time assay for cell proliferation and migration. Real-time assay for cell proliferation and migration were measured using an xCELLigence RTCA DP system (Roche Diagnostics, Indianapolis, IN, USA), which monitors cellular events in real-time without the incorporation of labels. Briefly, cells were placed into well of an E-plate 16 (for proliferation; U87-MG 2×10 ${ }^{4}$, U251-MG $1.2 \times 10^{4}$ cells) or Matrigel-coating (Matrigel: serum free media, 1:40) of the upper chamber of CIM-plate 16 (for migration; U87-MG 2x10², U251-MG $1.2 \times 10^{4}$ cells) and incubated for indicated times.

Statistical analysis. Data are expressed as the mean \pm SD from at least three separate experiments performed triplicate. The differences between groups were analyzed using a Student's t-test and $\mathrm{P}<0.05$ was considered significant, and $\mathrm{P}<0.01$ was highly significant compared with corresponding control values. Statistical analyses were carried out using SPSS software ver. 13.0 (SPSS, Inc., Chicago, IL, USA). Other statistical analysis performed using analysis of variance (ANOVA) and Tukey's post hoc tests, using the GraphPad Prism 5 software
(GraphPad Software, Inc., La Jolla, CA, USA). Differences were considered significant if $\mathrm{P}<0.05, \mathrm{P}<0.01$, and $\mathrm{P}<0.001$.

\section{Results}

TrioBP expression is high in glioblastoma tissue. Immunohistochemical (IHC) analysis using a tissue array and anti-TrioBP antibody showed stronger signals in brain tumor tissues than in surrounding normal tissues (Fig. 1A). Total lysates of normal and cancerous tissues from GBM patients were subjected to immunoblotting using an anti-TrioBP antibody. TrioBP levels in GBM tissues were higher than those in the surrounding normal tissues (Fig. 1B), suggesting that TrioBP expression is upregulated in glioblastoma patients.

TrioBP expression is elevated in glioblastoma cell lines. Based on the high TrioBP expression in samples from GBM patients (Fig. 1), TrioBP expression in glioblastoma cell lines was assessed by immunoblotting. TrioBP expression was significantly increased in U87-MG and U343-MG cells than in the other cell lines (Fig. 2A). Reverse transcription-quantitative polymerase chain reaction (RT-qPCR) showed that the mRNA level of TrioBP was increased in U343-MG, and U87-MG cells compared with that in the controls (Fig. 2B).

Upregulation of TrioBP $m R N A$ in U251-MG and U87-MG cells. As TrioBP expression was elevated in glioblastoma cells (Fig. 2A and B), the TrioBP mRNA levels in glioblastoma cell lines were determined by transcriptome profiling. Total RNA was prepared from U251-MG (low TrioBP expression) and U87-MG (high TrioBP expression) glioblastoma cells. Next, mRNA was split into small fragments to produce a cDNA library. The number of fragments per kilobase of exon per million fragments mapped (FPKM) was considered to be representative of the TrioBP mRNA level. FPKM values were greater in U87-MG cells (34.98) and U251-MG 


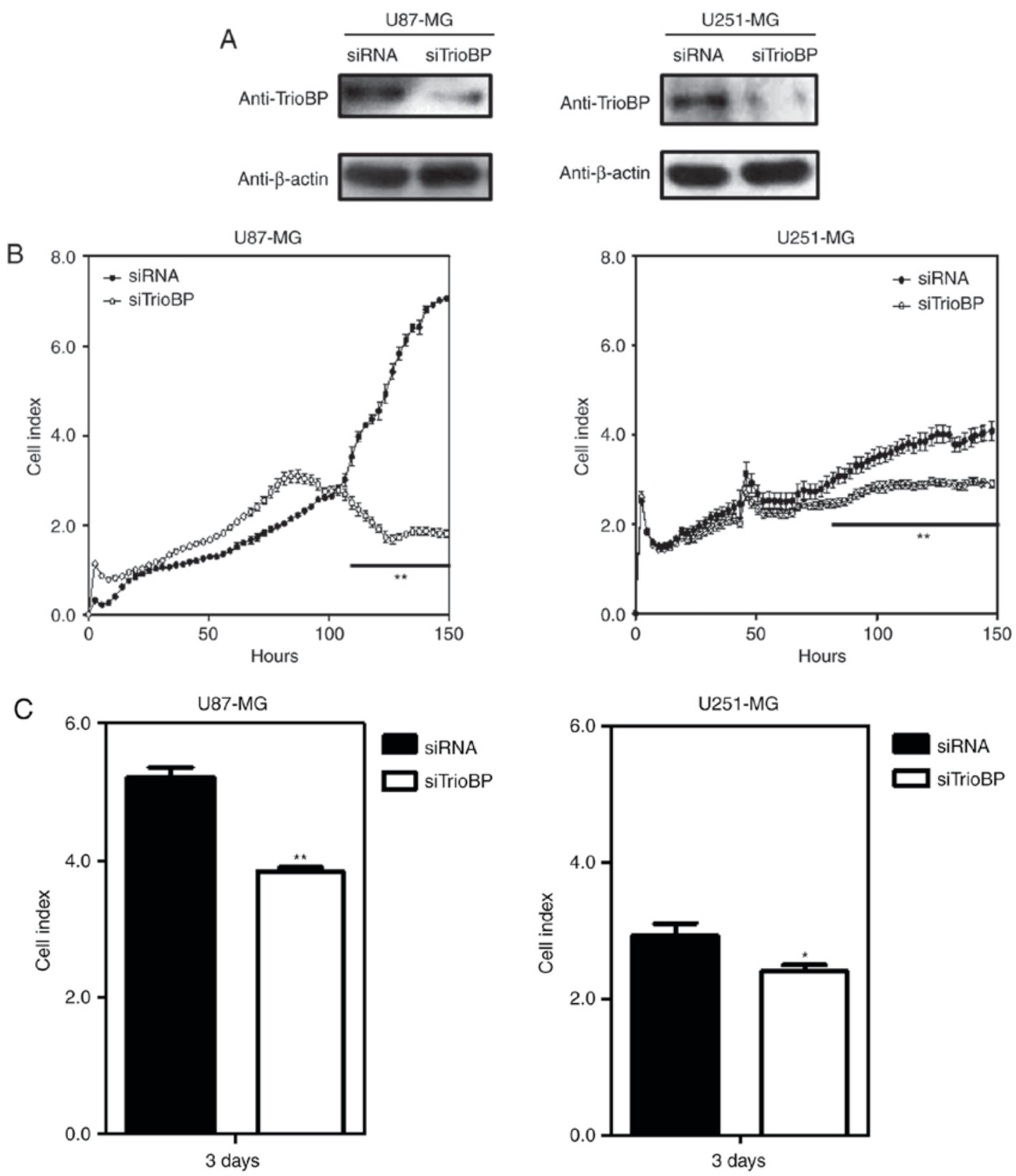

Figure 4. Effects of TiroBP on cell proliferation and migration in GBM cells. (A) U87 and U251-MG cells were transiently transfected with control-siRNA (siRNA) or TrioBP-siRNA (siTrioBP). After $72 \mathrm{~h}$-transfection, cell lysates were analyzed by western blotting with anti-TrioBP antibodies and anti- $\beta$-actin antibodies. (B) The proliferation of knockdowned cells were measured using an xCELLigence system in U87-MG cells (left panel) or U251-MG cells (right panel) for $150 \mathrm{~h}$. Values are the means \pm SEM $\left(\mathrm{n}=4 ;{ }^{* * *} \mathrm{P}<0.01\right)$. (C) The migration of knockdown cells were measured by an $\mathrm{xCELLigence} \mathrm{RTCA} \mathrm{DP} \mathrm{system}$ in U87-MG cells (left panel) and U251-MG cells (right panel) for $72 \mathrm{~h}$. These results are representative of three independent experiments $\left({ }^{*} \mathrm{P}<0.05,{ }^{* * *} \mathrm{P}<0.01\right)$.

cells (27.58) than in cerebral cortex cells (11.87) (Fig. 3), demonstrating that TrioBP transcription is enhanced in glioblastoma cells.

TrioBP knockdown suppresses the proliferation and migration of U251-MG and U87-MG cells. To investigate the roles of TrioBP in cancer cells, U251-MG and U87-MG cells were transfected with TrioBP siRNA and their proliferation and migration were evaluated by Excelligence. TrioBP expression was significantly reduced (Fig. 4A). siRNA-mediated knockdown of TrioBP inhibited the proliferation of U87-MG cells (left) and U251-MG cells (right) (Fig. 4B). The reduction in proliferation of U87-MG cells (left) was greater than that of U251-MG cells (right). In addition, the migration of U87-MG and U251-MG cells was suppressed (Fig. 4C). Therefore, TrioBP may play important roles in cancer cells.

Cytoplasmic localization of TrioBP in U251-MG cells. To investigate further its roles in GBM cells, the intracellular localization of TrioBP in U251-MG cells was evaluated. Interestingly, TrioBP was distributed in a punctate pattern, possibly associated with the cytoskeleton and cytosol (Fig. 5), indicating that localization to the cytosol is important for its function in glioblastoma cells.

Bioinformatic analysis of the TCGA GBM cohort. A bioinformatic analysis was performed on the TCGA GBM cohort. 

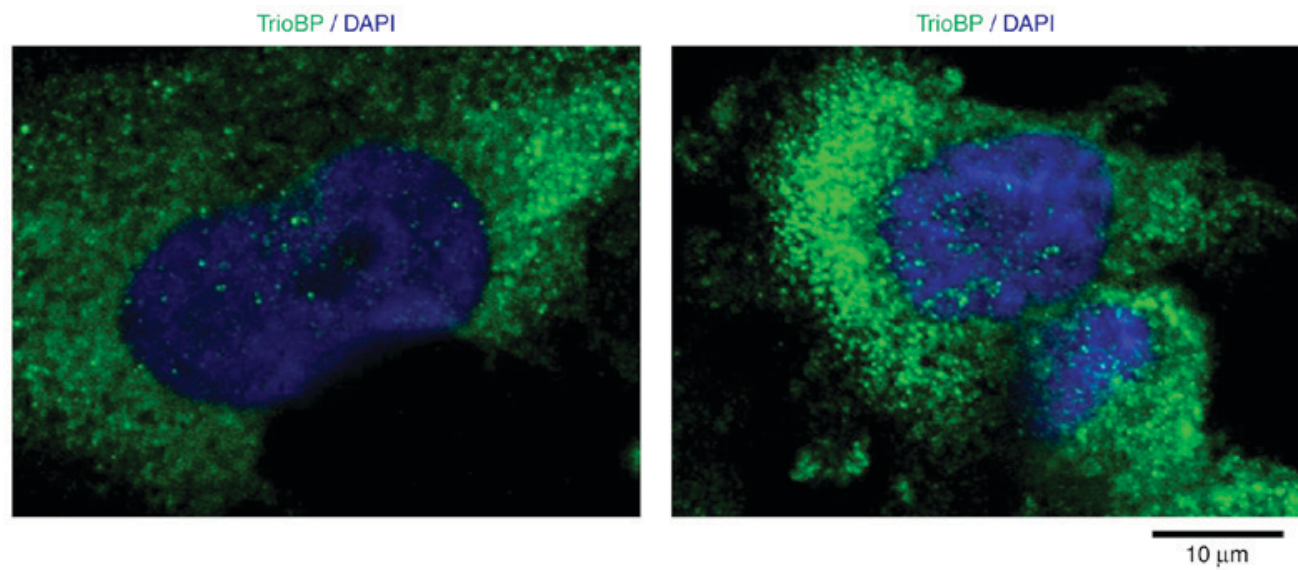

Figure 5. Intracellular localization of TrioBP in U251-MG cell U251-MG cells were grown on glass coverslips, fixed and permeabilized with $0.2 \%$ Triton X-100. After the immuno-staining of cells with anti-TrioBP antibody, cover slips were mounted with Vectashield and visualized using a Zeiss confocal microscope. Scale bars, $10 \mu \mathrm{m}$.

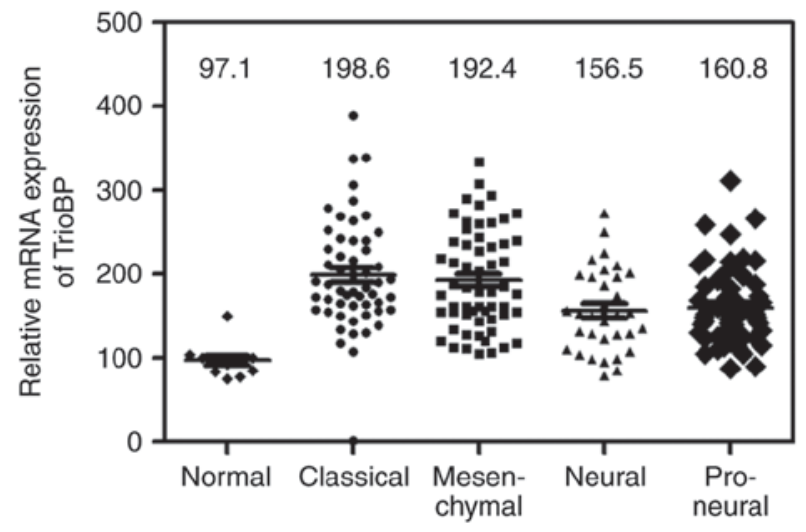

Figure 6. Bioinformatical analysis of glioma specimens for TrioBP mRNA expression from the TCGA GBM cohort The expression levels of TrioBP mRNA were detected in various glioblastoma (Normal; $\mathrm{n}=11$, Classical; $\mathrm{n}=54$, Mesenchymal; $\mathrm{n}=58$, Neural; $\mathrm{n}=33$, Proneural; $\mathrm{n}=57$ ). The median value of each group is indicated as a line.

Similar to the above results, TrioBP expression was increased in classical $(n=54)$, mesenchymal $(n=58)$, neural $(n=33)$, and proneural $(\mathrm{n}=57)$ glioblastomas compared with the level in normal controls $(n=11$; one-way analysis of variance, $\mathrm{P}<0.0001$; Fig. 6).

\section{Discussion}

GBM is an aggressive type of brain tumor with a poor prognosis despite the use of multiple therapies. Molecular targeting may facilitate the development of effective treatment strategies for GBM (1). Therefore, novel biomarkers and candidate therapeutics for glioblastomas are urgently needed.

In this study, TrioBP was found to have potential as a marker of GBM, as its expression was elevated in a GBM cell line and samples from GBM patients. Each of nine mutations in TrioBP leads to hearing loss, from prelingual severe to profound nonsyndromic deafness $(12,13)$. TrioBP interacts with Trio, which has rho guanine nucleotide exchange factor (GEF) activity due to its kinase domain (11), resulting in inhibition of the function of Trio in cell adhesion (22). RhoGEFs, downstream effectors of Rho GTPases, stimulate the invasiveness and survival of glioma cells (23). TrioBP-1 also interacts with F-actin, leading to the stabilization of F-actin structures (11). The characteristics of its binding partners (Trio and F-actin) $(23,24)$ suggest a role for TrioBP in tumorigenesis. However, the function of TrioBP in glioblastoma had not been investigated. Therefore, we determined whether TrioBP participates in the progression of brain tumors, including GBM. Unexpectedly, TrioBP expression was high in glioblastoma tissue (Fig. 1A). This was confirmed by immunoblotting of total cell lysates from normal and cancerous tissues from GBM patients sampled during surgery (Fig. 1B). TrioBP expression was also high in U87-MG and U343-MG cells (Fig. 2A). RT-qPCR analysis revealed that TrioBP mRNA levels are elevated in U343-MG, and U87-MG cells (Fig. 2B) compared with that in 293A cells. Furthermore, the FPKM of TrioBP mRNA level was elevated in U251-MG and U87-MG cells compared with that in normal brain cells (Fig. 3). TrioBP-specific siRNA in human mesenchymal stem cells suppresses cell proliferation and migration by decreasing F-actin levels (25). Moreover, TrioBP isoforms 4 and 5 reportedly promote the motility of pancreatic cancer cells (26). Indeed, the proliferation and migration (which are characteristics of GBM cells) of TrioBP-knockdown U87-MG and U251-MG cells were also inhibited (Fig. 4).

To investigate further its roles in GBM, the intracellular localization of TrioBP in U251-MG cells was evaluated. Interestingly, TrioBP was distributed in a punctate pattern, possibly associated with the cytoskeleton and cytosol (Fig. 5), indicating that localization to the cytosol is important for its function in glioblastoma cells. A bioinformatic analysis of the TCGA GBM cohort showed that TrioBP expression was high in all GBMs tested (Fig. 6), suggesting that TrioBP expression is correlated with the clinical parameters of gliomas. In conclusion, TrioBP is related to glioblastoma and may have potential for predicting the prognosis of glioblastoma patients.

\section{Acknowledgements}

This study was financially supported by research fund of Chungnam National University in 2015 (Jongsun Park) and by the National Research Foundation of Korea (NRF) grant funded 
by the Korea Government (MEST; NRF-2012M3A9B6055302, NRF-2014R1A1A3050752, NRF-2015R1A2A2A01003597 and NRF-2015R1D1A3A01015694).

\section{References}

1. Glaser T, Han I, Wu L and Zeng X: Targeted nanotechnology in glioblastoma multiforme. Front Pharmacol 8: 166, 2017.

2. Arif SH, Pandith AA, Bhat AR, Ramzan AU, Malik Nk, Chibber SS, Wani AA, Tabasum R and Altaf Kirmani A: EGFR and PTEN Gene Mutation Status in Glioblastoma Patients and their Prognostic Impact on Patient's Survival. J Carcinog Mutagen 6: 218, 2015.

3. Virk SM, Gibson RM, Quinones-Mateu ME and Barnholtz-Sloan JS: Identification of variants in primary and recurrent glioblastoma using a cancer-specific gene panel and whole exome sequencing. PLoS One 10: e0124178, 2015.

4. Bergers $\mathrm{G}$ and Benjamin LE: Tumorigenesis and the angiogenic switch. Nat Rev Cancer 3: 401-410, 2003.

5. Wesseling P, Ruiter DJ and Burger PC: Angiogenesis in brain tumors; pathobiological and clinical aspects. J Neurooncol 32: 253-265, 1997.

6. Das S and Marsden PA: Angiogenesis in glioblastoma. N Engl J Med 369: 1561-1563, 2013.

7. Carmeliet $P$ and Jain RK: Molecular mechanisms and clinical applications of angiogenesis. Nature 473: 298-307, 2011.

8. Norden AD, Drappatz J and Wen PY: Novel anti-angiogenic therapies for malignant gliomas. Lancet Neurol 7: 1152-1160, 2008

9. Chinot OL, Wick W, Mason W, Henriksson R, Saran F, Nishikawa R, Carpentier AF, Hoang-Xuan K, Kavan P, Cernea D, et al: Bevacizumab plus radiotherapy-temozolomide for newly diagnosed glioblastoma. N Engl J Med 370: 709-722, 2014.

10. Gilbert MR, Dignam JJ, Armstrong TS, Wefel JS, Blumenthal DT, Vogelbaum MA, Colman H, Chakravarti A, Pugh S, Won M, et al: A randomized trial of bevacizumab for newly diagnosed glioblastoma. N Engl J Med 370: 699-708, 2014.

11. Seipel K, O'Brien SP, Iannotti E, Medley QG and Streuli M: Tara, a novel F-actin binding protein, associates with the Trio guanine nucleotide exchange factor and regulates actin cytoskeletal organization. J Cell Sci 114: 389-399, 2001.

12. Shahin H, Walsh T, Sobe T, Abu Sa'ed J, Abu Rayan A, Lynch ED, Lee MK, Avraham KB, King MC and Kanaan M: Mutations in a novel isoform of TRIOBP that encodes a filamentous-actin binding protein are responsible for DFNB28 recessive nonsyndromic hearing loss. Am J Hum Genet 78: 144-152, 2006.

13. Riazuddin S, Khan SN, Ahmed ZM, Ghosh M, Caution K, Nazli S, Kabra M, Zafar AU, Chen K, Naz S, et al: Mutations in TRIOBP, which encodes a putative cytoskeletal-organizing protein, are associated with nonsyndromic recessive deafness. Am J Hum Genet 78: 137-143, 2006.
14. Lenz DR and Avraham KB: Hereditary hearing loss: From human mutation to mechanism. Hear Res 281: 3-10, 2011.

15. Kitajiri S, Sakamoto T, Belyantseva IA, Goodyear RJ, Stepanyan R, Fujiwara I, Bird JE, Riazuddin S, Riazuddin S, Ahmed ZM, et al: Actin-bundling protein TRIOBP forms resilient rootlets of hair cell stereocilia essential for hearing. Cell 141: 786-798, 2010.

16. Salhia B, Tran NL, Chan A, Wolf A, Nakada M, Rutka F, Ennis M, McDonough WS, Berens ME, Symons M and Rutka JT: The guanine nucleotide exchange factors trio, Ect 2 and Vav3 mediate the invasive behavior of glioblastoma. Am J Pathol 173: 1828-1838, 2008.

17. Kim HB and Yoo BS: Propolis inhibits UVA-induced Apoptosis of human keratinocyte HaCaT cells by scavenging ROS. Toxicol Res 32: 345-351, 2016

18. Li Y, Park J, Piao L, Kong G, Kim Y, Park KA, Zhang T, Hong J, Hur GM, Seok JH, et al: PKB-mediated PHF20 phosphorylation on Ser291 is required for p53 function in DNA damage. Cell Signal 25: 74-84, 2013.

19. Bioinformatics UG: The International Human Genome Sequencing Consortium. http://genome.ucsc.edu. Accessed 20: 2016, 2015.

20. Kim D and Salzberg S: TopHat 2.1.0. https://ccb.jhu.edu/software/tophat/index.shtml. Accessed July 29, 2015.

21. Na CH, Hong JH, Kim WS, Shanta SR, Bang JY, Park D, Kim HK and Kim KP: Identification of protein markers specific for papillary renal cell carcinoma using imaging mass spectrometry. Mol Cells 38: 624-629, 2015.

22. van Rijssel $J$ and van Buul JD: The many faces of the guanine-nucleotide exchange factor trio. Cell Adh Migr 6: 482-487, 2012.

23. Fortin Ensign SP, Mathews IT, Symons MH, Berens ME and Tran NL: Implications of Rho GTPase signaling in glioma cell invasion and tumor progression. Front Oncol 3: 241, 2013.

24. Li A, Dawson JC, Forero-Vargas M, Spence HJ, Yu X, König I, Anderson $\mathrm{K}$ and Machesky LM: The actin-bundling protein fascin stabilizes actin in invadopodia and potentiates protrusive invasion. Curr Biol 20: 339-345, 2010.

25. Yun SP, Ryu JM, Jang MW and Han HJ: Interaction of profilin-1 and F-actin via a $\beta$-arrestin-1/JNK signaling pathway involved in prostaglandin $\mathrm{E}(2)$-induced human mesenchymal stem cells migration and proliferation. J Cell physiol 226: 559-571, 2011.

26. Bao J, Wang S, Gunther LK, Kitajiri S, Li C and Sakamoto T: The actin-bundling protein TRIOBP-4 and -5 promotes the motility of pancreatic cancer cells. Cancer Lett 356: 367-373, 2015.

(i) (5) This work is licensed under a Creative Commons Attribution-NonCommercial-NoDerivatives 4.0 International (CC BY-NC-ND 4.0) License. 